\title{
LTP Induction Translocates Cortactin at Distant Synapses in Wild-Type But Not Fmr1 Knock-Out Mice
}

\author{
Ronald R. Seese, ${ }^{1 \star}$ Alex H. Babayan, ${ }^{1 *}$ Adam M. Katz, ${ }^{1}$ Conor D. Cox,${ }^{1}$ Julie C. Lauterborn, ${ }^{1}$ Gary Lynch, ${ }^{1,2}$ \\ and Christine M. Gall ${ }^{1,3}$ \\ Departments of ${ }^{1}$ Anatomy and Neurobiology, ${ }^{2}$ Psychiatry and Human Behavior, and ${ }^{3}$ Neurobiology and Behavior, University of California, Irvine, \\ California 92697-1275
}

\begin{abstract}
Stabilization of long-term potentiation (LTP) depends on reorganization of the dendritic spine actin cytoskeleton. The present study tested whether this involves activity-driven effects on the actin-regulatory protein cortactin, and whether such effects are disturbed in the Fmr1 knock-out (KO) model of fragile X syndrome, in which stabilization of both actin filaments and LTP is impaired. LTP induced by theta burst stimulation (TBS) in hippocampal slices from wild-type mice was associated with rapid, broadly distributed, and NMDA receptor-dependent decreases in synapse-associated cortactin. The reduction in cortactin content was blocked by blebbistatin, while basal levels were reduced by nocodazole, indicating that cortactin's movements into and away from synapses are regulated by microtubule and actomyosin motors, respectively. These results further suggest that synapse-specific LTP influences cytoskeletal elements at distant connections. The rapid effects of TBS on synaptic cortactin content were absent in Fmr1 KOs as was evidence for activity-driven phosphorylation of the protein or its upstream kinase, ERK1/2. Phosphorylation regulates cortactin's interactions with actin, and coprecipitation of the two proteins was reduced in the KOs. We propose that, in the KOs, excessive basal phosphorylation of ERK1/2 disrupts its interactions with cortactin, thereby blocking the latter protein's use of actomyosin transport systems. These impairments are predicted to compromise the response of the subsynaptic cytoskeleton to learning-related afferent activity, both locally and at distant sites.
\end{abstract}

\section{Introduction}

Newly induced long-term potentiation (LTP) passes through a 10-15 min period during which the potentiated state becomes extremely persistent, a process requiring the assembly and subsequent stabilization of spine filamentous (F) actin (Krucker et al., 2000; Rex et al., 2009). Recent work determined that signaling involving the GTPase RhoA and the actin-severing protein cofilin is critical for the formation of new filaments (Rex et al., 2009), but the events underlying their delayed stabilization are poorly understood. Clues came with the observation that theta burst stimulation (TBS) commonly used to produce LTP activates $\mathrm{p} 21$ activated kinase (PAK) and Src tyrosine kinases (Chen et al., 2007, 2010a). PAK and Src target cortactin (Lua and Low, 2005), a protein that promotes $\mathrm{F}$-actin elaboration and stabilization in many cellular systems. Cortactin activates the Arp2/3 complex, initiates F-actin branching, and stabilizes branch sites (Mullins et al., 1998; Uruno et al., 2001; Weaver et al., 2001). Cortactin also

Received Aug. 8, 2011; revised Feb. 28, 2012; accepted March 29, 2012.

Author contributions: R.R.S., A.H.B., J.C.L., G.L., and C.M.G. designed research; R.R.S., A.H.B., A.M.K., and J.C.L. performed research; R.R.S., A.H.B., C.D.C., J.C.L., G.L., and C.M.G. analyzed data; R.R.S., A.H.B., J.C.L., G.L., and C.M.G. wrote the paper.

This work was supported by NIH Grants MH082042 and NS045260. R.R.S. was supported in part by Medical Scientist Training Program Grant T32-GM08620. We thank Dr. Christopher S. Rex and Dr. Lulu Y. Chen for valuable contributions leading to the present study, and Andrew Wang, Jihua Liu, and Yue Qin Yao for technical assistance.

*R.R.S. and A.H.B. contributed equally to this work.

The authors declare no competing financial interests.

Correspondence should be addressed to Christine M. Gall, Department of Anatomy and Neurobiology, University of California, Irvine, Irvine, CA 92697-1275. E-mail: cmgall@uci.edu.

DOI:10.1523/JNEUROSCI.0968-12.2012

Copyright $\odot 2012$ the authors $\quad 0270-6474 / 12 / 327403-11 \$ 15.00 / 0$ prevents F-actin depolymerization by capping barbed ends (Le Clainche et al., 2007) and inhibiting cofilin (Oser et al., 2009). As expected from this, cortactin regulates and maintains dendritic spine morphology in cultured neurons (Hering and Sheng, 2003). Together, these findings suggest that cortactin may contribute to the production and stabilization of changes in spine anatomy that occur with LTP (De Roo et al., 2008; Yang et al., 2008).

Cortactin's influence on actin networks and morphology in nonneuronal cells depends on movement between membrane and cytoplasmic compartments. Notably, events associated with LTP-activation of synaptic brain-derived neurotrophic factor and glutamate receptors - cause cortactin to move into and out of spines in dissociated neurons, respectively (Hering and Sheng, 2003; Iki et al., 2005; Chen and Hsueh, 2012). PAK and ERK1/2 regulate cortactin mobility; whether actomyosin motors, which contribute importantly to LTP (Rex et al., 2010), are involved is not known. Accordingly, initial goals of the present study were to test whether naturalistic TBS influences cortactin levels at hippocampal synapses and whether such effects involve active transport mechanisms.

Actin regulation is also of interest with regard to spine abnormalities in neuropsychiatric disorders (Penzes et al., 2011). Aberrant spine morphology is assumed to reflect defects in the underlying cytoskeleton, which could arise from reduced effects of synaptic activity on actin networks. In support of this, LTP does not properly consolidate in rodent models of several human conditions associated with abnormal spines (Lynch et al., 2008). For the Fmr1 knock-out (KO) model of fragile $\mathrm{X}$ syndrome, 
which is characterized by poorly developed spines (Irwin et al., 2000), the consolidation deficit is associated with impaired actinregulatory signaling through Rac GTPase (Chen et al., 2010b). Prompted by these findings, we also evaluated TBS effects on cortactin in adult Fmr1 KO hippocampus.

Results show that induction of LTP reduces cortactin concentrations at wild-type (WT) synapses, within and beyond fields of synaptic activation. These effects were profoundly disturbed in Fmr1 KOs, likely reflecting impairments in ERK1/2 activation and processes that associate cortactin with local transport machinery. Disruption of synaptic activity effects on cytoskeletal regulatory sequences likely contributes to disturbances in spine morphology, synaptic plasticity, and cognitive function in the mutants.

\section{Materials and Methods}

Animals. Fmr1 KO mice backcrossed onto the FVB-129 background for 11 generations (Irwin et al., 2002; Lauterborn et al., 2007) and WT agematched FVB mice were used. Procedures were conducted in accordance with NIH guidelines and approved by the Institutional Animal Care and Use Committee of the University of California, Irvine.

Electrophysiology. Acute transverse hippocampal slices (350 $\mu \mathrm{m}$ thick) were prepared from adult (2- to 4-month-old) male Fmr1 KO and WT mice and 2-month-old male Sprague Dawley rats using a Leica Vibroslicer as described previously (Lauterborn et al., 2007) with some modifications. The Vibroslicer cutting bath included artificial CSF (ACSF) with high $\mathrm{Mg}^{2+}$ [containing (in $\mathrm{mm}$ ) $124 \mathrm{NaCl}, 3 \mathrm{KCl}, 1.25 \mathrm{KH}_{2} \mathrm{PO}_{4}, 5$ $\mathrm{MgSO}_{4}, 26 \mathrm{NaHCO}_{2}$, and 10 dextrose, $\left.\mathrm{pH} 7.4\right]$ or, when specified, choline chloride [containing (in $\mathrm{mM}$ ) 110 choline chloride, $2.5 \mathrm{KCl}, 1.25$ $\mathrm{NaH}_{2} \mathrm{PO}_{4}, 5 \mathrm{MgSO}_{4}, 25 \mathrm{NaHCO}_{2}, 25$ glucose, 11.6 ascorbic acid, and 3.1 pyruvic acid, pH 7.4] (Li et al., 2009). Slices were, in all cases, transferred to a holding chamber containing high- $\mathrm{Mg}^{2+}$ ACSF during the cutting process and then to an interface recording chamber set to $31^{\circ} \mathrm{C}$ with constant perfusion of ACSF containing the following (in mM): $124 \mathrm{NaCl}$, $3 \mathrm{KCl}, 1.25 \mathrm{KH}_{2} \mathrm{PO}_{4}, 2.5 \mathrm{CaCl}_{2}, 1.5 \mathrm{MgSO}_{4}, 26 \mathrm{NaHCO}_{2}$, and $10 \mathrm{dex}-$ trose, $\mathrm{pH}$ 7.4. Slices were equilibrated in the chamber for $2 \mathrm{~h}$ before use.

For induction of LTP, stimulation electrodes were placed in stratum (str.) radiatum of fields CAla and CAlc, and a field recording electrode was placed in CA1b str. radiatum; the two stimulation electrodes drove distinct populations of synapses in the recording field and were used to increase the numbers of synapses activated with TBS. Stimulation was set to elicit field EPSPs (fEPSPs) that were $50 \%$ of the maximum population spike-free amplitude. After stable baseline fEPSPs were collected for 20 min, TBS (10 bursts of four pulses at $100 \mathrm{~Hz} ; 200 \mathrm{~ms}$ between bursts) or control stimulation (three single pulses per minute) was applied at each stimulation electrode (with the two stimulation trains separated by $30-40 \mathrm{~s}$ ); stimulus pulse duration was increased to $1.5 \times$ baseline settings during TBS only. Test responses to three pulses per minute at baseline strength were then collected until tissue harvest. For antidromic stimulation experiments, one stimulating electrode was placed in the alveus (superficial axon bundles) on the subicular side of the recording electrode, which was located in the pyramidal cell layer of CA1b. Following a 10 min baseline, slices were stimulated using control stimulation (three pulses per minute) or TBS and then recorded for another $5 \mathrm{~min}$. The stimulating electrode was then moved to a second alvear site; baseline was again recorded followed by either control stimulation or TBS. Slices were harvested 10 min later.

In some experiments, the myosin II kinase inhibitor blebbistatin (10 $\mu \mathrm{M}$; Tocris Bioscience), the microtubule inhibitor nocodazole (200 nм; Tocris Bioscience), or the NMDA receptor antagonist DL-2-amino-5phosphonopentanoic acid (APV; $100 \mu \mathrm{m}$; Tocris Bioscience) was infused into the tissue bath via a second perfusion line for $\sim 30-60 \mathrm{~min}$; the compounds remained in the bath until tissue harvest $7 \mathrm{~min}$ following stimulation.

Immunohistochemistry. As described previously (Chen et al., 2010b), hippocampal slices were harvested into cold $4 \%$ paraformaldehyde, subsectioned at $20 \mu \mathrm{m}$ using a freezing microtome, and slide mounted. The tissue was incubated overnight at room temperature in a primary antisera mixture containing mouse antisera to postsynaptic density-95 (PSD-95; 1:1000; Thermo Scientific, catalog \#MA1-054) or goat antisera to GluR1 (1:100; Santa Cruz Biotechnology, catalog \#sc-7609) in combination with rabbit antisera to cortactin (1:250; Santa Cruz Biotechnology, catalog \#sc-11408), phosphorylated (p)-cortactin Ser405 (1:500; Protea Bio, catalog \#AB-100), or p-ERK1/2 Thr202/Tyr204 (1:500; Cell Signaling Technology, catalog \#4370). After washes in $0.1 \mathrm{M}$ phosphate buffer (PB), pH 7.2, the tissue was incubated for $1 \mathrm{~h}$ at room temperature in a mixture of Alexa Fluor 488 anti-mouse $\operatorname{IgG}$ or anti-goat $\operatorname{IgG}$ with Alexa Fluor 594 anti-rabbit IgG (1:1000 each; Invitrogen). Primary and secondary antibodies were diluted in $0.1 \mathrm{M} \mathrm{PB}$ containing $4 \% \mathrm{BSA}$ and $0.3 \%$ Triton $\mathrm{X}-100$. After final $\mathrm{PB}$ rinses, the tissue was coverslipped with VectaShield (Vector Labs).

For experiments evaluating the incidence of dendritic spine immunoreactivity for cortactin and p-cortactin, brains of adult (2- to 4-monthold) male WT and Fmr1 KO mice were fast frozen in 2-methylbutane $\left(-50^{\circ} \mathrm{C}\right)$ and cryostat sectioned on the coronal plane at $20 \mu \mathrm{m}$. The slide-mounted tissue sections were fixed in $-20^{\circ} \mathrm{C}$ methanol for $20 \mathrm{~min}$, washed in $0.1 \mathrm{M} \mathrm{PB}$, and then processed for dual immunofluorescence as described above.

Image acquisition and analysis. For hippocampal slice experiments, three to four tissue sections through each slice were used to acquire image $z$ stacks ( $0.2 \mu \mathrm{m}$ steps) of immunolabeling within the CAlb str. radiatum recording field (between the stimulating electrodes) using a Leica DM6000 epifluorescence microscope. For fast-frozen brain sections, image $z$ stacks were collected (as above) from CAlb str. radiatum of three to four tissue sections through midseptotemporal hippocampus. In both instances, images were processed for iterative deconvolution (Volocity 4.1 Restorative Deconvolution; PerkinElmer). Automated in-house software was then used to construct three-dimensional montages of the $136 \times 105 \times 3 \mu \mathrm{m}$ sample fields (Rex et al., 2009; Chen et al., 2010b) to count and measure volumes of single- and double-labeled puncta within the size constraints of dendritic spines. Puncta were counted as double labeled if there was any overlap in their immunolabeled boundaries as evaluated in three dimensions. Counts of double-labeled PSDs were normalized to the total number of PSD-95-immunopositive $(+)$ puncta within a given sample field. Tissue sections with cortactin + PSD counts that were more than 4 SDs removed from the mean of the remainder of the sample fields from that group (all sections for all slices in a group) were not included in the analysis. The aberrant section values tended to occur on particular slices, resulting in a loss of $3.4 \%$ of the slices. Section exclusions because of the 4 SD rule for the remainder of the slices were $4.9 \%$.

Synaptoneurosome and Western blot analysis. Male and female WT and Fmr1 KO mice, at 16-18 d of age, were deeply anesthetized with isoflurane and decapitated. Forebrains were isolated, homogenized in a glass tube with Teflon pestle, and centrifuged at $2500 \times g$ to remove contaminating erythrocytes. Synaptoneurosomes were isolated at the interface of a two-phase (7.5 and 10\%) Ficoll gradient following ultracentrifugation at $28,000 \times \mathrm{g}$ for $50 \mathrm{~min}$ at $4^{\circ} \mathrm{C}$ (Bernard-Trifilo et al., 2005). Samples were adjusted to $1.5 \mu \mathrm{g} / \mu \mathrm{l}$ using ACSF and then either (1) immunoprecipitated (Thermo Scientific Co-Immunoprecipitation Kit) with anticortactin (Santa Cruz Biotechnology, catalog \#sc-30772) followed by Western blot analysis or (2) run immediately for 8,10 , or $12 \%$ PAGE Western blot analysis (Rex et al., 2009). In all cases, blots were incubated overnight at $4^{\circ} \mathrm{C}$ in primary antisera including mouse antisera to actin (1:10,000; Sigma, catalog \#A1978) and p-ERK1/2 Thr202/Tyr204 (1: 1500; Cell Signaling Technology, \#9106) and rabbit antisera to ERK1/2 (1:2500; Cell Signaling Technology, catalog \#9102), PAK1 (1:100; Cell Signaling Technology, catalog \#2602), PAK3 (1:100; Millipore, catalog \#06-902), and cortactin (1:2000; Santa Cruz Biotechnology, catalog \#sc11408). Bands were visualized using ECL + chemiluminescence (GE Healthcare), quantified using Image $(\mathrm{NIH})$, and normalized to sample actin or input-cortactin content.

Statistical analyses. Statistical significance (considered as $p<0.05$ ) was assessed using a two-way repeated-measures ANOVA with planned post hoc comparisons, a two-tailed Student's $t$ test, or the Mann-Whitney $U$ test. A single $n$ was considered to be a hippocampal slice for immunohis- 
tochemical analyses following electrophysiology, an animal for immunohistochemical analyses of brain tissue sections, and an independently treated sample in studies with synaptoneurosomes. Unless otherwise specified, values in text and figures show group means \pm SEM.

\section{Results}

Electron microscopy (EM) has produced somewhat conflicting results regarding the localization of cortactin within spines. Preembedding immuno-EM analyses described two pools, a small one near the PSD and a larger pool located deeper within the spine head (Racz and Weinberg, 2004). These experiments found that only a small percentage of synapses are associated with high levels of the protein. Other work using high-pressure freezing EM indicated that nearly all cortactin is distributed within $100 \mathrm{~nm}$ of the cytoplasmic face of the synapse (Rostaing et al., 2006), but this study did not address variations in cortactin immunoreactivity (IR) across synapses or types of spines. Both works found that cortactin was present at low to negligible levels in axon terminals. Given the uncertainties about localization, we used rigorous criteria to identify postsynaptic elements associated with cortactin IR. First, cortactin-immunopositive puncta were counted as being associated with a synapse only when they overlapped, as evaluated in three dimensions, elements immunoreactive for the integral PSD protein, PSD-95 (Walikonis et al., 2000; Aoki et al., 2001) (Fig. 1A). Second, to identify cortactinenriched synapses, we restricted the analysis to cortactin clusters whose labeling intensity was $\geq 85 \%$ above the mean for control slices. Using these criteria, 1-6\% of the PSD-95+ elements that met the size and eccentricity constraints for synapses were colocalized with dense concentrations of cortactin in CA1 str. radiatum of acute hippocampal slices. PSDs (and concentrations of PSD-95) are exclusively localized to asymmetric, spine synapses in adult rodent str. radiatum (Aoki et al., 2001; Megías et al., 2001); thus, these values reflect numbers of spines containing high concentrations of cortactin IR in the sample field.

\section{Theta burst stimulation decreases cortactin + synapses in WT hippocampus}

To test for effects of TBS on spine cortactin content, WT hippocampal slices received either one train of 10 theta bursts or control low-frequency (three pulses per minute) stimulation delivered to two stimulating electrodes placed in the apical Schaffer collateral-commissural projections to field CA1b. Slices were harvested at a range of time points from 7 to $90 \mathrm{~min}$ after TBS and processed for dual immunofluorescence localization of PSD-95 and cortactin; the numbers of double-labeled puncta were quantified for the $105 \times 136 \times 3 \mu \mathrm{m}$ sample field, and values from slices receiving TBS were normalized to measures from yoked (same mouse), low-frequency controls.

TBS caused a pronounced decrease in the number of PSD$95+$ elements double labeled for dense cortactin IR in WT slices; the effect was significant over the period from 7 to $45 \mathrm{~min}$ after TBS, with numbers returning to control levels by $90 \mathrm{~min}$ after stimulation (Fig. $1 B$, left). We repeated the experiment using a modified ACSF (during cutting only) that contained choline chloride $(110 \mathrm{~mm})$ instead of $\mathrm{NaCl}$; in agreement with previous reports (Kirov et al., 2004), we find that removal of $\mathrm{NaCl}$ better preserves dendritic morphology and spine numbers through slice preparation at low temperatures (A. H. Babayan, unpublished observations). In the choline chloride-prepared slices, TBS caused a $\sim 50 \%$ decrease in the number of densely cortactin+ PSDs (Fig. $1 B$, right). We further tested for the reduction using the AMPA receptor subunit GluR1 as a marker for excitatory
A
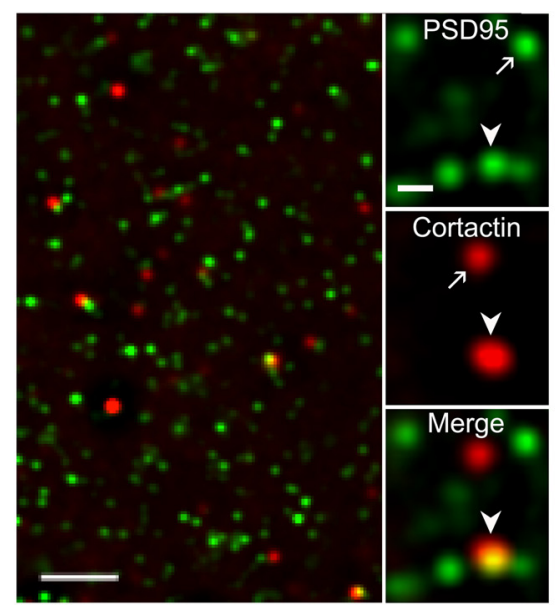

B
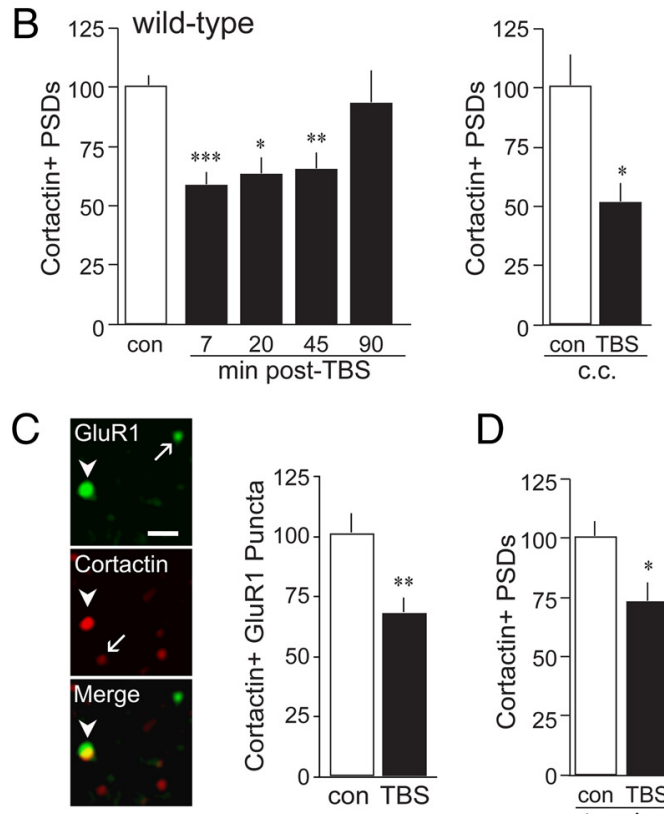

D

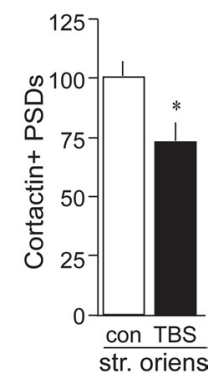

Figure 1. TBS decreased spine cortactin content in WT slices. Slices received control three pulses per minute stimulation [control (con)] or TBS to the apical Schaffer-commissural afferents to field CA1 and were processed for dual immunofluorescence localization of cortactin and PSD-95. A, Left, Low-power deconvolved photomicrograph shows merged immunolabeling for PSD-95 and cortactin in the CA1b field of analysis in hippocampus from a WT mouse. Scale bar, $2.5 \mu \mathrm{m}$. Right, High-power photomicrographs show punctate immunoreactivity for PSD-95 and cortactin (and merged) after iterative deconvolution in CA1 str. radiatum. Scale bar, $1 \mu \mathrm{m}$. Arrows and arrowheads indicate single- and double-labeled puncta, respectively. $\boldsymbol{B}$, Bar graphs show numbers of double-labeled puncta in the CA1 sample field; in this and subsequent panels, counts are expressed as a percentage of mean control values. Left, TBS (black bars, values normalized to those from paired controls) decreased numbers of cortactin + PSDs in str. radiatum of WT slices from 7 to 45 min after stimulation ( $n \geq 7$ per group). Right, TBS applied to slices cut into choline chloride (c.c.)-containing ACSF also decreased cortactin + PSDs in str. radiatum as assessed 7 min after TBS ( $n \geq 3$ per group). C, Left, Adult WT slices were stimulated as above, harvested at $7 \mathrm{~min}$ after TBS, and processed for dual-immunofluorescence localization of cortactin and the AMPA receptor subunit GluR1. Photomicrographs show punctate immunoreactivity for both markers after iterative deconvolution in CA1 str. radiatum. Arrows and arrowheads indicate single- and double-labeled elements, respectively. Scale bar, $1 \mu \mathrm{m}$. Right, Bar graph shows that the number of densely cortactin-immunoreactive elements colocalized with GluR1 + clusters fulfilling the size and eccentricity constraints of synapses is decreased at 7 min after TBS (black bar) in CA1 str. radiatum ( $n=10$ per group). $\boldsymbol{D}$, TBS was applied to apical Schaffer-commissural projections; slices processed for PSD-95 and cortactin localization, and previously evaluated for apical field labeling at $20 \mathrm{~min}$ after TBS $(\boldsymbol{B})$, were photographed for quantitative analyses in the basal dendritic field (str. oriens). TBS significantly decreased numbers of cortactin + PSDs in CA1b str. oriens ( $n \geq 8$ per group). ${ }^{*} p<0.05$; ${ }^{* *} p<0.01$; ${ }^{* * *} p<$ 0.001 versus control. 

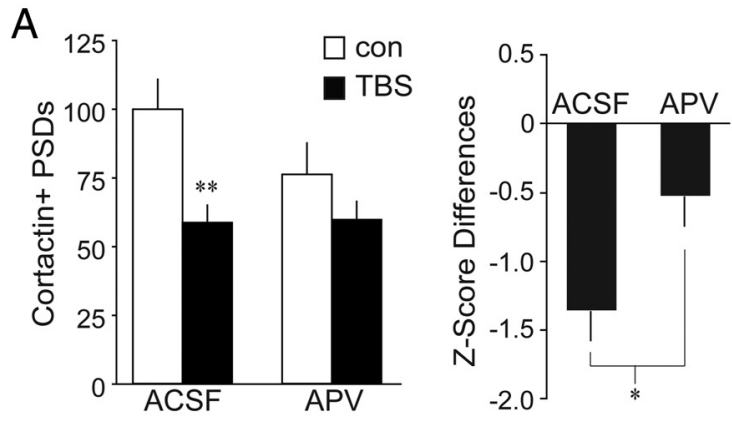

B
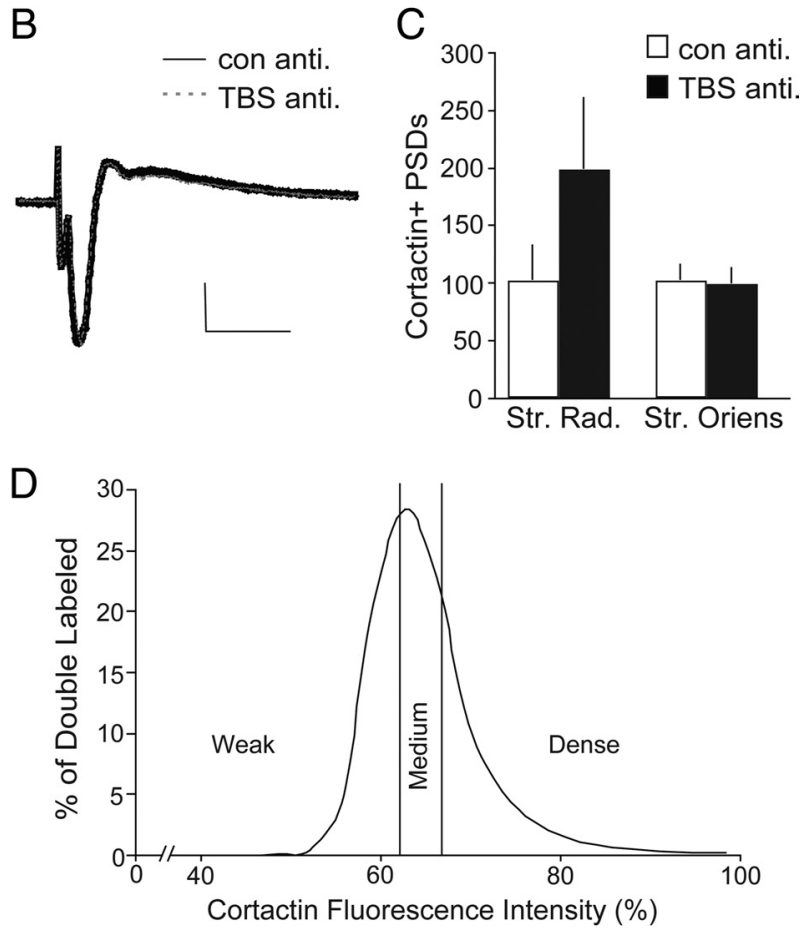

$E$

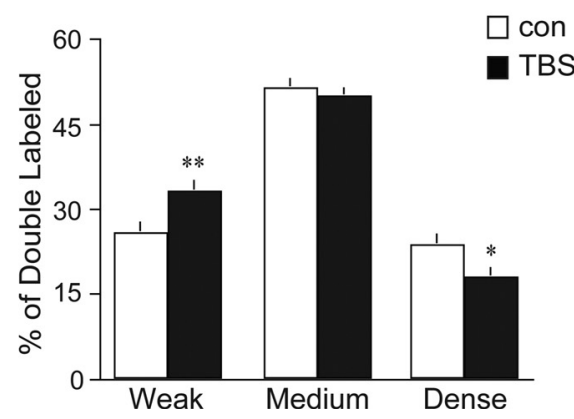

Figure 2. The TBS-induced reduction in synaptic cortactin is NMDA receptor-dependent and evident across the broad fluorescence intensity distribution. $\boldsymbol{A}$, Left, Hippocampal slices from adult WT mice received TBS or control (con) stimulation and were harvested $7 \mathrm{~min}$ later for dual immunofluorescence localization of cortactin and PSD-95 in CA1b str. radiatum. TBS (black bars) reduced numbers of densely cortactin + PSDs in WT slices bathed in normal ACSF (values are expressed as a percentage of paired ACSF controls; ${ }^{* *} p<0.01$ vs ACSF controls); this reduction was blocked in slices treated with the NMDA receptor antagonist APV (100 $\mu \mathrm{m})$ for $30 \mathrm{~min}$ before stimulation ( $n \geq 6$ per group). Right, Bar graph shows $Z$ score differences for doublelabeled element counts in both TBS groups (ACSF or APV) compared to the mean of each group's respective controls (value for each TBS slice minus the mean of the no-TBS group, divided by SD of the no-TBS group). TBS effects on cortactin + PSDs were significantly smaller in the presence of APV compared to those in normal ACSF $\left({ }^{*} p=0.016\right)$. $B$, Representative antidromic (anti.) population spikes, recorded in CA1 pyramidal cells following alvear stimulation, were unaffected by theta bursts. Calibration: $1 \mathrm{mV}, 5 \mathrm{~ms}$. C, Bar graph shows quantification of cortactin + PSDs following control antidromic or theta burst antidromic stimulation. Antidromic TBS synapses. Using the same size and eccentricity criteria described above for recognizing synapses, we found that $1.84 \pm 0.49 \%$ (mean $\pm \mathrm{SD}$ ) of the synapse-sized GluR1 clusters were tightly associated with dense concentrations of cortactin IR in slices administered only control stimulation. TBS reduced this doublelabeled value to $1.24 \pm 0.32 \%$ (mean \pm SD) of the total synapse population, a $32 \%$ reduction from control that was highly significant $(p<0.005)$ (Fig. 1C).

TBS activates only a small percentage of the synapses in the apical CA1 dendritic field (Chen et al., 2007), and it is therefore unlikely that the substantial losses of synaptic cortactin IR described here are restricted to those contacts directly affected by TBS. Analyses of the CA1b basal dendrites, which are not innervated by the stimulated str. radiatum projections (Amaral and Lavenex, 2007), confirmed this point: theta bursts reduced the number of PSDs associated with dense cortactin IR in str. oriens by about $30 \%$ (i.e., to $72.8 \pm 8.3 \%$ of control; $p<0.05$ ) (Fig. $1 D$ ). To corroborate this finding, the laminar distribution of TBS effects was evaluated in hippocampal slices from adult rat. Theta bursts delivered to str. radiatum caused a decrease in synapses colocalized with dense concentrations of cortactin in str. oriens of rat slices as assessed $7 \mathrm{~min}$ after stimulation (control, $4.8 \pm 0.7 \%$; TBS, $2.1 \pm 0.3 \%$; expressed as a percentage of the total PSD-95+ population; $p=0.01$ for four slices per group).

We next asked whether the LTP-related events set in motion by TBS are involved in the loss of cortactin labeling at synapses scattered across much of the pyramidal cell's dendritic tree. WT slices were pretreated with the NMDA receptor antagonist APV for 30 min before TBS and then collected 7 min after stimulation. Matched controls were infused with APV but did not receive TBS; an additional two groups (control stimulation vs TBS) were tested in the absence of the NMDA receptor antagonist. TBS again markedly $(-41 \%)$ reduced the number of densely cortactin + synapses in slices maintained in normal ACSF $(p<0.007)$ but failed to produce a statistically significant effect in APVtreated slices $(-22 \% ; p>0.20)$ (Fig. $2 A$, left). Interpretation of these results is complicated by a tendency for APV to reduce the percentage of double-labeled synapses in control (three pulses per minute stimulation only) slices (Fig. $2 A$ ); while this effect did not approach significance $(p=0.15)$, it could conceivably obscure any losses produced by TBS. We therefore converted the percentage of doubled-labeled synapses in the two TBS groups to $Z$ scores for differences from the means of their respective controls (value for each TBS slice minus the mean of the no-TBS group divided by the SD of the no-TBS group). TBS produced a reduction of $-1.30 \pm 0.50$ from matched controls for slices maintained in control ACSF and a $-0.50 \pm 0.58$ decrease in APV-treated slices (means \pm SDs; $p=0.016$ ) (Fig. $2 A$, right). We conclude that blocking NMDA receptors has a marked and

$\leftarrow$

elicited a nonsignificant $(p=0.22$ ) increase in double-labeled counts in str. radiatum (Str. Rad.) with no change in number of cortactin + PSDs in the str. oriens (basal dendritic field; values are expressed as a percentage of yoked slices receiving antidromic control stimulation; $n \geq 5$ per group). $D$, Curve shows the cortactin immunofluorescence intensity distribution for cortactin/ PSD-95 double-labeled elements in field CA1b of WT slices receiving control stimulation. $\boldsymbol{E}$, Bar graph of the same results plotted in $\boldsymbol{D}$ shows the number of double-labeled elements with cortactin + labeling intensities falling within the bottom 25\% (weak labeling), middle 50\% (medium labeling), and top 25\% (dense labeling) of values from control slices. TBS caused a significant shift in the distribution of elements across these categories (2-way ANOVA, $p=$ 0.0002 ), decreasing the intensely cortactin-immunolabeled population with a concomitant increase in the weakly immunolabeled population. ${ }^{*} p<0.05$; ${ }^{* *} p<0.01$ vs respective control ( $n \geq 21$ per group). 
negative effect on TBS-driven changes in the number of densely cortactin + synapses.

How does theta-driven NMDA receptor activation, in a small set of synapses within a discrete portion of the dendritic tree, produce cell-wide changes in cortactin concentrations at nonactivated contacts? A likely explanation involves the induction of action potentials at cell bodies followed by the retrograde propagation of spikes into the proximal apical and basal branches of the neuron's dendritic arborization. We tested this idea using TBS-driven antidromic spiking of CA1 pyramidal cells. A stimulating electrode was placed in the alveus (superficial axon bundles) on the subicular side of a recording electrode lowered into the CA1b pyramidal cell layer (identified visually and by cell spiking), and TBS was delivered after baseline recording. This was repeated for two alvear sites per slice. Single stimulation pulses produced a large (3-7 $\mathrm{mV}$ ) antidromic population spike (Fig. $2 B$ ) that was much larger than any recorded during orthodromic TBS. As expected, the size of the antidromic responses was not altered by theta bursts (Fig. $2 \mathrm{~B}$ ). Normalization to matched controls showed that antidromic TBS had no measurable effect on the densely cortactin + synapses in str. oriens and caused a nonsignificant increase in str. radiatum (Fig. 2C). In all, cell spiking in the theta burst pattern does not reduce the number of cortactin+ synapses in hippocampal field CA1.

Collectively, the above results suggest that an event initiated by the prolonged depolarization and calcium increases produced by orthodromic TBS, but not by much briefer cell spiking episodes, spreads extensively along dendrites and affects a significant number of cortactin-rich synapses.

The above analyses dealt with synapses with dense immunostaining for cortactin ( $\geq 85 \%$ above the mean immunolabeling intensity for control slices). The marked reductions produced by TBS could therefore reflect an effect on a small population of synapses with particularly high levels of the protein or a TBSinduced leftward shift of the curve relating numbers of colabeled synapses to the intensity of cortactin labeling, thereby affecting a markedly larger population of synapses. We distinguished between these alternatives by constructing a curve relating cortactin immunolabeling intensity to the number of double-labeled contacts for control slices and then dividing it into the bottom 25\% (weak cortactin labeling), the middle 50\% (medium labeling), and the top 25\% (dense labeling) (Fig. 2D). The distribution of cortactin + contacts across these intensity categories was clearly changed by TBS (Fig. $2 E$ ) (two-way ANOVA, $p=0.0002$ ): the decrease in densely labeled synapses in the TBS group was accompanied by an equivalent increase in the proportion of synapses with weak cortactin immunostaining. Importantly, the total number of double-labeled synapses was unchanged by TBS (control, 14,034 \pm 582 per sample zone; TBS, 13,364 \pm 827 ), as was the total number of PSD-95+ elements $(30,978 \pm 1156$ vs $30,218 \pm 1559)$. We conclude from these calculations that TBS reduces cortactin concentrations in a substantial percentage of the synaptic population.

\section{Effects of TBS on synaptic cortactin are impaired in Fmr1 KO slices}

Prior studies showed that TBS increases dendritic spine levels of activated Rac and p-PAK Ser141 in field CA1 of hippocampal slices from WT but not Fmr1 KO mice (Chen et al., 2010b). Both Rac and PAK modulate cortactin compartmentalization (Weed et al., 2000; Webb et al., 2006), suggesting that cortactin movement within spines might be impaired in the mutants. Analysis of immunofluorescence within brain tissue sections from adult
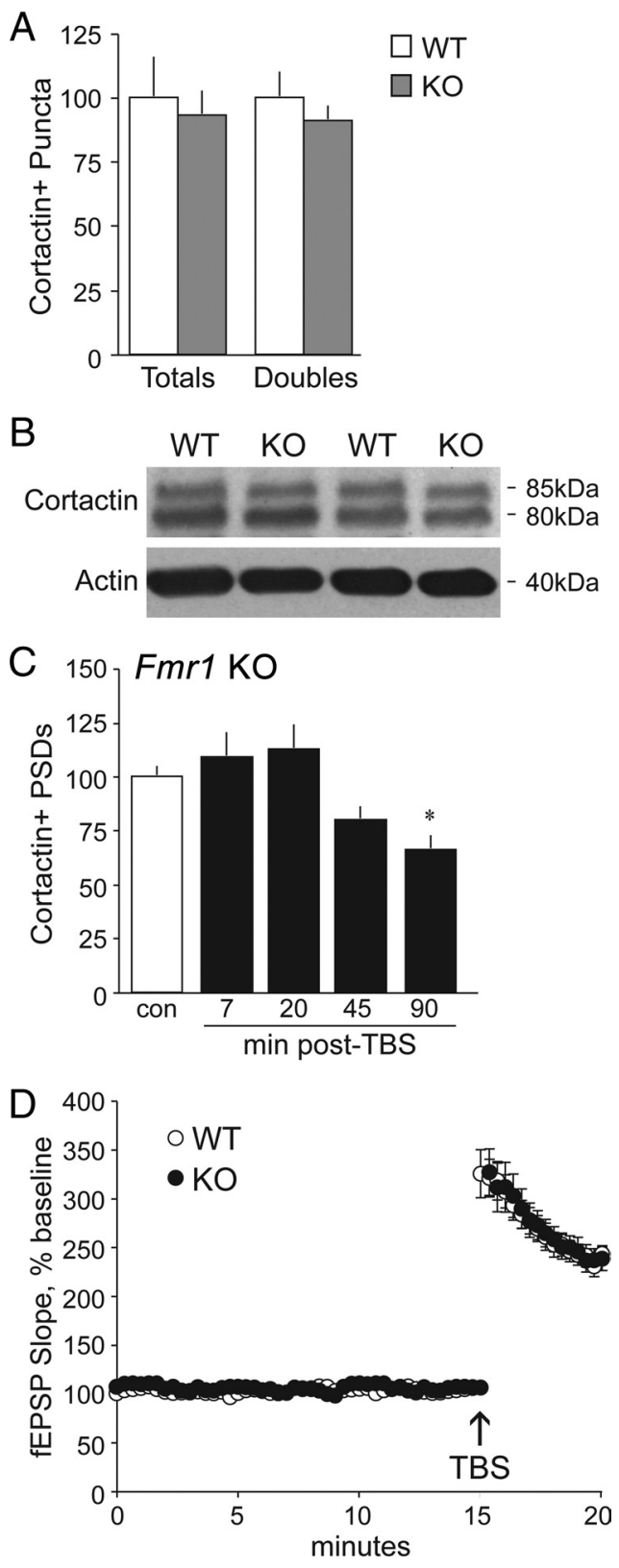

Figure 3. TBS-induced changes in spine cortactin content are severely delayed in Fmr $1 \mathrm{KO}$ s A, Quantification of cortactin + puncta (Totals) and those double labeled for PSD-95 (Doubles) demonstrate no differences between WT (white bars) and Fmr $1 \mathrm{KO}$ (gray bars) male mice, as assessed in field CA1b str. radiatum (values are expressed as a percentage of WT). $\boldsymbol{B}$, Representative Western blot shows comparable levels of cortactin immunoreactivity (at 85 and $80 \mathrm{kDa}$ ) in synaptoneurosomal samples from WT and Fmr 1 KO mice. C, Quantification of cortactin + PSDs in CA1 str. radiatum in Fmr $1 \mathrm{KO}$ slices that received control (con) stimulation or TBS (black bars, values normalized to paired controls) shows that the theta train significantly decreased numbers of cortactin + PSDs at 90 min after TBS only ( ${ }^{*} p<0.05$ vs control; $n \geq 7$ per group). $\boldsymbol{D}$, Plot shows fEPSP slopes collected before and after TBS applied to slices from WT and KO mice (for each slice, mean \pm SEM values are expressed as the percentage of the mean baseline fEPSP slope; $n=8$ per group); the magnitude of potentiation was comparable in the two genotypes through 20 min after stimulation.

male Fmr1 KO and WT mice detected no effect of genotype on numbers of densely cortactin + puncta double labeled for PSD-95 within CA1 str. radiatum (WT, $4.44 \pm 0.45 \%$; KO, $4.05 \pm 0.26 \%$; expressed as a percentage of the total PSD-95+ population; $p=0.46$, two-tailed Student's $t$ test) (Fig. $3 A$ ). Moreover, total cortactin IR in synaptoneurosomal samples did not 
differ between genotypes as determined by Western blot analysis (Fig. $3 B$ ). Nonetheless, and in marked contrast to the results from WT slices, TBS had no effect on the number of PSDs associated with dense cortactin IR in Fmr1 KO mice through the first 20 min after stimulation; numbers trended lower by $45 \mathrm{~min}$, and were significantly below control values at $90 \mathrm{~min}$ after TBS (Fig. 3C). The markedly delayed effect of TBS on synaptic cortactin IR in the KOs is not likely to reflect differences in EPSP responses to the theta train. As described previously (Lauterborn et al., 2007), input/output curves, fEPSP waveforms, and composite responses to the theta bursts are comparable in slices from FVB WT and Fmr1 KO mice. We also found that short-term potentiation and the initial expression of LTP following a 10-burst theta train are indistinguishable between WT and Fmr1 KO slices (Lauterborn et al., 2007). We replicated the latter findings in slices harvested here for immunostaining at later post-TBS time points (Fig. 3D). Together, these results indicate that impaired activity-dependent changes in cortactin content at Fmr1 KO synapses involve defects in the cellular events set in motion by theta stimulation rather than genotype differences in levels of depolarization achieved with TBS.

\section{Active transport maintains cortactin levels at synapses in WT mice}

The TBS-induced loss and recovery of synaptic cortactin in WT slices could reflect proteolysis followed by the arrival of new protein copies or the operation of translocation processes. To distinguish between these possibilities, and potentially understand the nature of defects in the Fmr1 KOs, we applied blebbistatin, a potent and selective toxin that inhibits myosin II function and thus activation of myosin motors (Straight et al., 2003). As demonstrated in prior work with hippocampal slices (Rex et al., 2010), the dose of blebbistatin used here has no detectable effect on field CA1 baseline fEPSPs, paired-pulse facilitation, mEPSCs, the input/output curve generated with single pulse stimulation, or theta burst responses, but it does completely block LTP. In the present study, we further determined that blebbistatin infused for 30-60 min has no effect on the mean size (Fig. 4A) or size frequency distribution (data not shown) of PSD-95+ elements in CA1b str. radiatum. Nonetheless, blebbistatin completely eliminated TBS-induced decreases in cortactin IR associated with PSDs in hippocampal slices from WT mice (Fig. 4B).

Spines contain significant levels of tubulin, and microtubule treadmilling is hypothesized to contribute to protein transport between spines and dendritic shafts (Gu and Zheng, 2009; Jaworski et al., 2009). Moreover, cortactin reportedly associates with the positive end of microtubules in spines (Jaworski et al., 2009). We tested for a microtubule role in regulating spine cortactin levels by acutely treating slices with low concentrations of nocodazole (200 nM, $1 \mathrm{~h}$ ), which blocks polymerization of the dynamic populations of microtubules normally found in spines (Vasquez et al., 1997; Jaworski et al., 2009). The results were surprising: nocodazole had no effect on the size (Fig. 4C) or waveforms (Fig. $4 D$ ) of fEPSPs, or on the total number of PSD$95+$ elements (data not shown), but caused a $\sim 35 \%$ decrease in the number of synapses associated with dense concentrations of cortactin IR in WT slices $(p<0.05)$ (Fig. $4 E$ ). We next asked whether the labile pool of cortactin IR maintained at synapses by microtubule-dependent processes overlaps that reduced by TBS. Nocodazole was infused beginning $1 \mathrm{~h}$ before delivering theta stimulation. Under these conditions, TBS did not detectably change the number of densely cortactin + synapses from that found in matched WT slices that received control stimulation as
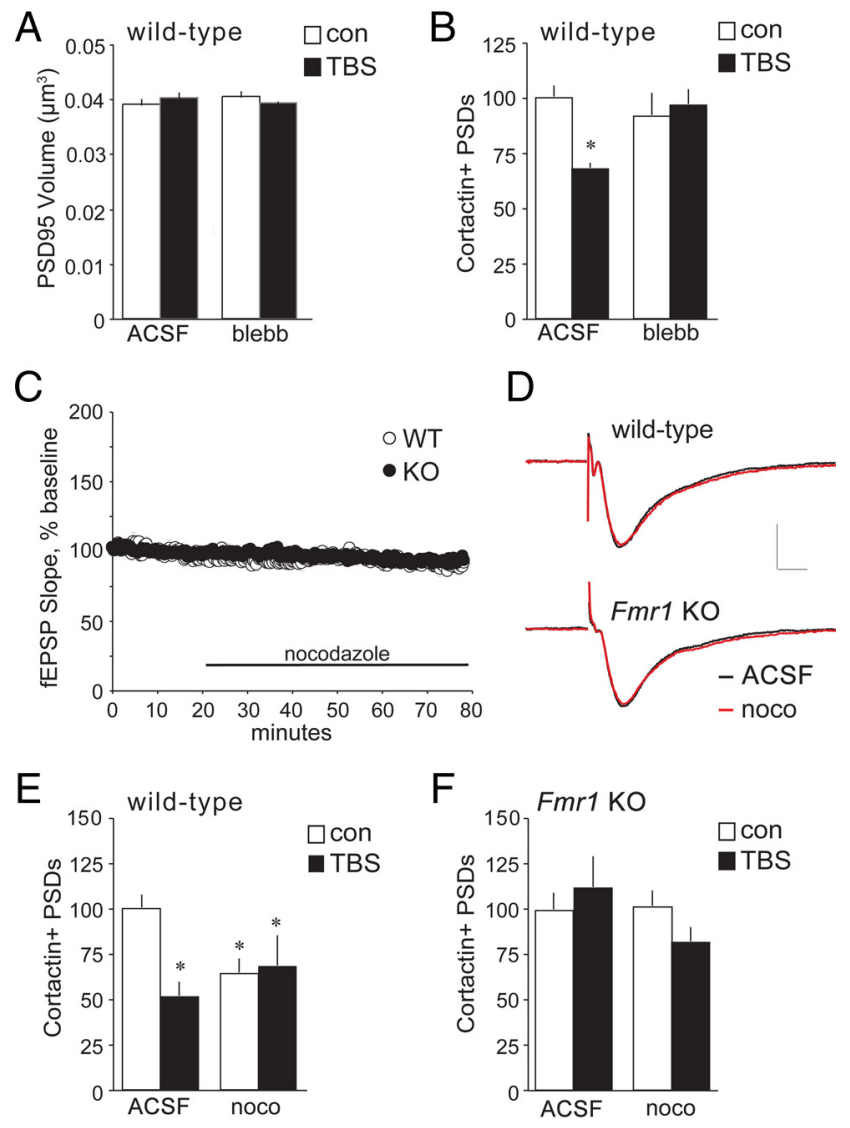

Figure 4. Actomyosin and microtubule motors regulate spine cortactin content. Hippocampal slices from WT and Fmr1 KO mice received TBS or control (con) stimulation and were harvested $7 \mathrm{~min}$ later for immunofluorescence analysis of cortactin and PSD-95 double labeling in CA1b str. radiatum. $\boldsymbol{A}$, Bar graph showing no difference in volumes of PSD-95+ elements between slices treated with the myosin II kinase inhibitor (S)-(-)-blebbistatin (blebb; $10 \mu \mathrm{m}$ ) for $1 \mathrm{~h}$ from those bathed in normal ACSF, regardless of stimulation paradigm. $\boldsymbol{B}$, TBS (black bars) reduced numbers of cortactin + PSDs in WT slices bathed in normal ACSF (values normalized to paired ACSF controls; ${ }^{*} p<0.05$ vs ACSF con); this reduction was blocked in slices treated with (S)-(-)-blebbistatin (10 $\mu \mathrm{m} ; n \geq 6$ per group). $C$, Treatment with nocodazole (noc0; 200 $\mathrm{nm}, 1 \mathrm{~h}$ ) did not significantly affect baseline fEPSP responses in WT or Fmr1 K0 slices (mean \pm SEM values are shown; $n=4$ per group). $D$, Representative fEPSP traces, collected before (black trace) and after (red trace) nocodazole infusion, show no treatment effect on waveforms in slices from WT (top) or Fmr 1 KO (bottom) mice. Calibration: $1 \mathrm{mV}, 5 \mathrm{~ms}$. $\boldsymbol{E}$, Bar graphs show that in WT slices, nocodazole reduced numbers of cortactin + PSDs in control slices (white bars) and prevented further reductions with TBS (black bars; values normalized to paired ACSF controls; ${ }^{*} p<0.05$ vs WT ACSF control; $n=7$ per group). $\boldsymbol{F}$, In slices from Fmr $1 \mathrm{KO}$ mice, neither TBS nor nocodazole influenced numbers of cortactin + PSDs ( $n=8$ for K0 controls; $n=3$ for KO-TBS).

assessed at $7 \mathrm{~min}$ (Fig. $4 \mathrm{E}$ ). In marked contrast to its actions in WT slices, nocodazole did not reduce the baseline number of double-labeled synapses in Fmr1 KO cases; as in untreated mutant slices, TBS had no effect on numbers of densely cortactin+ contacts (Fig. $4 F$ ).

Collectively, the above results point to the conclusion that WT synapses have a dynamic pool of cortactin that is both continuously replenished by a microtubule-dependent mechanism and rapidly diminished by TBS via actomyosin motors. The fragile $\mathrm{X}$ mutation appears to block both of these cortactin-related transport-dependent processes.

\section{Cortactin's phosphorylation and actin interactions are deficient in Fmr1 KO hippocampus}

A failure to properly interact with actin-based transport systems could account for the observed disturbance of TBS effects on 
A
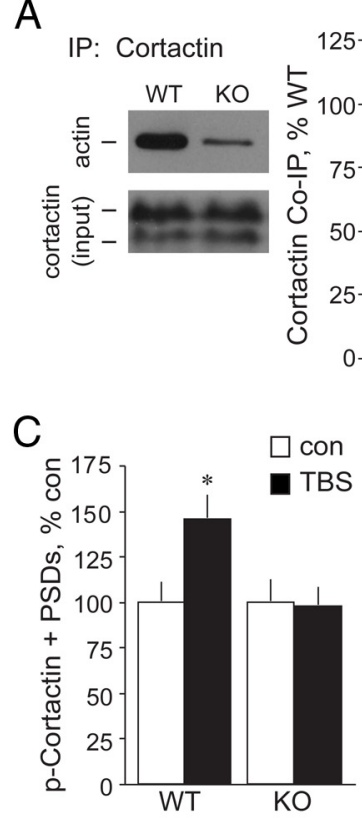
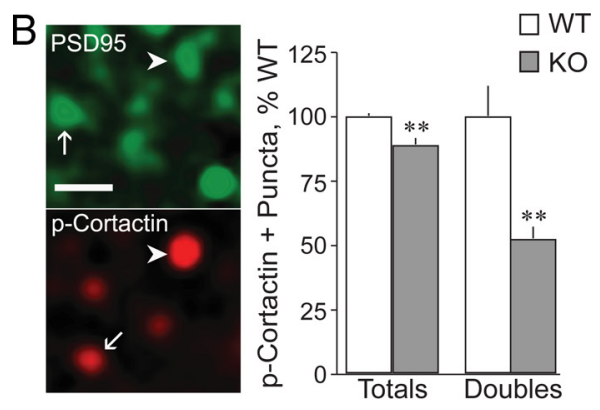

within the cell (Martinez-Quiles et al., 2004; Iki et al., 2005; Cosen-Binker and Kapus, 2006; Kruchten et al., 2008). We tested for genotype effects on cortactin Ser405 phosphorylation at synapses in CA1 str. radiatum in brain tissue sections using dual immunostaining and found the number of densely phosphorylated cortactin + PSDs to be reduced by $\sim 50 \%$ in Fmrl KOs relative to WTs (Fig. 5B). Further evidence that kinase signaling to cortactin is disturbed in the mutants was apparent after TBS. A single train of 10 theta bursts produced a robust and reliable increase in the number of PSDs associated with high concentrations of p-cortactin in slices from WT mice, but had no effect in Fmrl KOs (Fig. 5C). These results indicate that phosphorylation defects are likely contributors to the reduced association of cortactin with F-actin in the mutants.

We next asked whether changes to up-

$E$

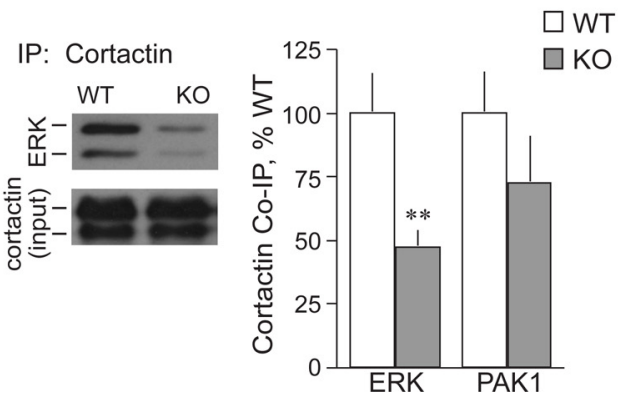

$\mathrm{F}$

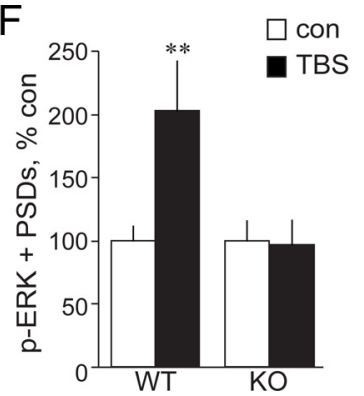

Figure 5. Cortactin's serine phosphorylation and association with actin are impaired in $F m r 1 \mathrm{KO}$ synapses. A, Left, Representative Western blots of WT and Fmr $1 \mathrm{KO}$ synaptoneurosomal samples immunoprecipitated with anti-cortactin and then probed for actin (band at $\sim 40 \mathrm{kDa}$ ). The bottom blot shows comparable input cortactin levels (before precipitation). Right, Quantification of actin-immunoreactive bands from blots of cortactin immunoprecipitates (normalized to input cortactin levels) shows significantly reduced association of actin with cortactin in K0 synaptoneurosomes (gray bars; ${ }^{* *} p<0.01$ vs WT; $n \geq 7$ per group; in $A, B, D$, and $\boldsymbol{E}$, the K0 measures are expressed as percentages of WT values). $\boldsymbol{B}$, Left, Photomicrographs show punctate immunoreactivity for p-cortactin (Ser405) and PSD-95 in CA1 str. radiatum; arrows and arrowheads indicate single- and double-labeled puncta, respectively. Scale bar, $1 \mu \mathrm{m}$. Right, Counts of total p-cortactin (Ser405)-immunopositive puncta (Totals) and those double-labeled for PSD-95 (Doubles) demonstrate that within K0s (gray bars), compared to WTs (white bars), field CA1 numbers of p-cortactin + puncta are lower, most particularly within the PSD-95+ postsynaptic compartment ${ }^{* *} p<0.01$ vs WT). C, TBS (black bars) increases counts of $p$-cortactin + PSDs in WT, but not K0, slices [values normalized to paired controls (con); ${ }^{*} p<0.05$ vs WT control; $n \geq 8$ per group]; in $\boldsymbol{C}$ and $\boldsymbol{F}$, values from TBS groups are expressed as percentages of those from the same-genotype control means. $\boldsymbol{D}$, Left, Representative Western blots of WT and $F m r 1 \mathrm{KO}$ forebrain synaptoneurosomal samples probed for $\mathrm{p}$-ERK (Thr202/Tyr204; bands at 44 and 42 kDa). Blot of actin illustrates consistent loading. Right, Quantification of p-ERK (Thr202/ Tyr204) IR, divided by sample actin IR, shows robust hyperphosphorylation of ERK in the Fmr $\mathrm{KO}$ synaptic fractions (gray bars) compared to WT samples (values normalized to WT; ${ }^{* *} p<0.01 ; n \geq 8$ per group). Total synaptic ERK content is comparable between the two genotypes ( $p=0.15 ; n=5$ per group). $\boldsymbol{E}$, Left, Representative Western blots of WT and Fmr 1 KO synaptoneurosomal samples immunoprecipitated with anti-cortactin and then probed for ERK1/2 (bands at 44 and $42 \mathrm{kDa}$ ). The bottom blot shows comparable input cortactin levels. Right, Quantification of ERK-immunoreactive (44 kDa) and PAK1-immunoreactive bands from blots of cortactin immunoprecipitates (normalized to input cortactin levels) shows significantly reduced association of ERK with cortactin in K0 synaptoneurosomes (gray bars; values normalized to WT; ${ }^{* *} p<0.01$ vs WT; $n \geq 4$ per group). $\boldsymbol{F}$, TBS (black bars) increases numbers of PSDs associated with p-ERK (Thr202/Tyr204) in WT, but not K0, field CA1 (values normalized to paired controls; ${ }^{* *} p<0.01$ vs WT control; $n \geq 5$ per group).

synaptic cortactin concentrations in Fmrl KO slices. We tested this using coimmunoprecipitation from synaptoneurosomes. Levels of total actin and cortactin immunoreactivities were comparable in samples prepared from forebrains of Fmrl KO and WT mice, but $\sim 40 \%$ less actin coimmunoprecipitated with cortactin in the mutants (Fig. 5A). Serine phosphorylation regulates cortactin's interactions with F-actin and its ability to translocate this prediction: immunoprecipitation of cortactin brought down much less ERK immunoreactivity in synaptoneurosomes from KOs compared to WTs (Fig. 5E). There was a trend for reduced coprecipitation of PAK1 with cortactin in the KOs, but this did not approach statistical significance (Fig. $5 E)$. Similarly, coprecipitation of PAK3 with cortactin was not significantly different in samples from KO and WT mice ( $p=$ 0.27 ; data not shown). 
The hyperphosphorylation of ERK in the mutants would also be expected to disturb any response of the kinase to TBS. We tested this and found that TBS doubled the number of PSDs colocalized with p-ERK in WT slices while having no detectable effect in the mutants (Fig. 5F). Collectively, results described in this section suggests a hypothesis in which abnormal phosphorylation of ERK leads to (1) a baseline reduction in cortactin phosphorylation in the synaptic compartment, resulting in (2) reduced association of the protein with actomyosin motors and (3) a subsequent failure of TBS to translocate cortactin away from synapses.

\section{Discussion}

The present results demonstrate that TBS causes rapid reductions in cortactin levels at excitatory synapses in adult hippocampus of WT, but not Fmr1 KO, mice. Although intense NMDA receptor activation has been shown to cause cortactin redistribution from spines into the dendritic shaft (Hering and Sheng, 2003; Iki et al., 2005), our studies provide novel evidence that naturalistic activity patterns used to induce LTP elicit rapid, activity-driven, and motor-dependent movement of the protein away from synapses in adult brain. Moreover, the distribution of changes in synaptic cortactin IR indicates that the effect is not limited to contacts activated by TBS.

A sizable body of work indicates that LTP is synapse specific, an observation critical to the argument that the effect is a substrate for memory. The present results challenge the synapse specificity of at least some of the neurochemical changes associated with LTP. Specifically, we show that potentiation of a subpopulation of synapses in CA1 str. radiatum reduces cortactin levels at distant synapses, including those within str. oriens. This suggests that localized LTP affects how distant contacts will respond to subsequent afferent activity. Without challenging the synapse specificity of LTP, prior work has shown that potentiation in one population of synapses initiates protein synthesisdependent events that lower the threshold for modification in neighboring synapses (i.e., synaptic "tagging") (Alarcon et al., 2006; Reymann and Frey, 2007). Cortactin translocation could represent part of the tagging machinery but, as the shift occurs within minutes of stimulation, it is likely to cause changes well in advance of protein synthesis-dependent events.

The spatially extended effects of TBS were detected by evaluating PSDs associated with dense cortactin IR in particular, an approach that facilitated quantitative analysis of TBS-induced changes. However, analysis of the broad cortactin immunofluorescence intensity distribution showed that TBS shifts the distribution of synaptic cortactin + clusters from "dense cortactin IR" to "weak cortactin IR" categories without changing the total number of double-labeled contacts. Thus, cortactin did not vacate spines, as has been reported to occur in cultured cells with intense NMDAR stimulation. It will be of interest in future research to determine whether delivery of TBS to multiple sites on target neurons, as opposed to the single apical sublamina used here, reduces cortactin levels in a greater proportion of synapses. This could have functional consequences that do not necessarily violate the synapse specificity of LTP. As discussed, cortactin regulates the architecture and stability of the actin cytoskeleton, and these effects reflect its disposition across subcellular compartments (Weed et al., 2000; Oser et al., 2009). Since cytoskeletal reorganization is critical to the stabilization, but not induction or initial expression, of LTP (Lynch et al., 2008; Ramachandran and Frey, 2009), we propose that widespread effects on cortactin compartmentalization represent a form of metaplasticity (Pérez-

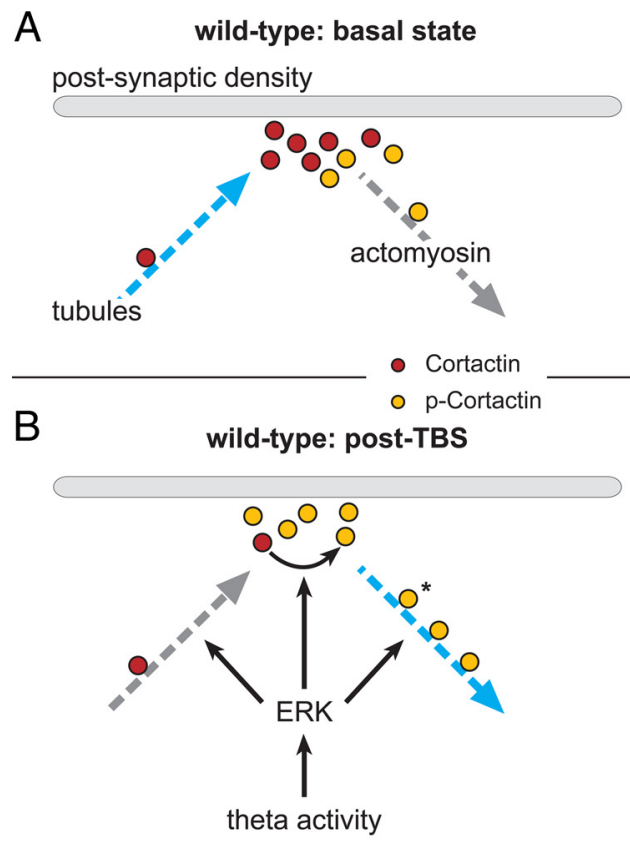

\section{Fmr1 KO: basal state and post-TBS}

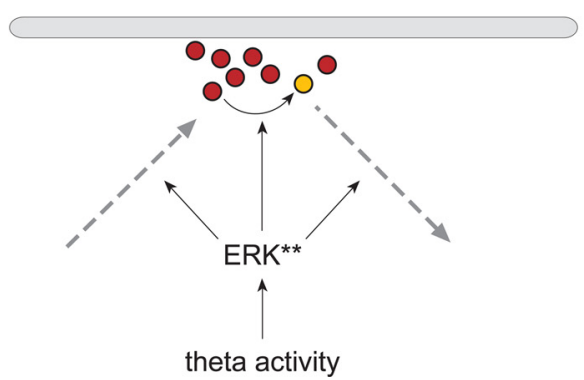

Figure 6. Actomyosin motors and microtubules mediate synaptic cortactin motility, and fragile X model mice underutilize both systems. $A$, At WT synapses in the basal state, synaptic cortactin concentrations (red-filled circles) are maintained by nocodazole-sensitive, microtubule mechanisms (blue arrow). $\boldsymbol{B}$, Following TBS, synaptic ERK and cortactin are phosphorylated (orange-filled circles, p-cortactin). These synaptic signaling modifications are accompanied by a pronounced, blebbistatin-sensitive decrease in synaptic cortactin levels, indicating that naturalistic afferent activity uses myosin motors (blue arrow) to actively translocate cortactin away from the PSD. *The illustrated association and motility of p-cortactin with actomyosin motors are based on reports that cortactin's phosphorylation mediates its association with actin. Thus, it is proposed that following TBS, cortactin is first phosphorylated and then transported away from the PSD. C, In Fmr 1 KO mice, basal synaptic ERK is hyperphosphorylated $\left.{ }^{* *}\right)$ and completely unresponsive to changes in afferent drive (thin arrows). As a result, in the mutants, ERK fails to associate with and phosphorylate cortactin, thereby substantially decreasing synaptic $p$-cortactin levels and disrupting both cortactin's association with actin and thetadriven movement away from PSDs (dashed arrow, right). Moreover, abnormalities in phosphorylation of ERK, a kinase that also regulates microtubule dynamics, may account for the finding that constitutive cortactin levels at the PSD are not maintained by a nocodazolesensitive transport system (dashed arrow, left) in the KOs.

Otaño and Ehlers, 2005) that affects serial consolidation events set in motion by the arrival of TBS at previously naive connections. Consolidation both maintains LTP (Lynch et al., 2007; Bramham, 2008) and increases its resistance to reversal by lowfrequency stimulation (Stäubli and Scafidi, 1999; Kramár and Lynch, 2003). Thus, tests for reversibility could be used to determine whether induction of localized LTP affects the stability of subsequently induced potentiation at distant synapses in a cortactin-dependent fashion. In all, the present study raises the 
possibility that learning-related afferent activity elicits neuronwide changes that influence the later production of enduring synaptic changes.

Our results place constraints on mechanisms likely to mediate TBS effects on synaptic cortactin levels. Theta-driven antidromic spikes had no significant effects on numbers of densely cortactin + PSDs, suggesting that backpropagation of action potentials into dendrites is not involved. However, the NMDA receptor antagonist APV blocked the interaction between TBS and synaptic cortactin content. Possibly, repetitive and prolonged (50-100 ms) episodes of depolarization produced by TBS (Larson and Lynch, 1988; Chen et al., 2010a; zur Nedden et al., 2011), or elevations of local calcium concentrations that follow NMDA receptor activation, initiate biochemical events that spread for some distance into the dendritic tree. A first step in discriminating between the depolarization versus calcium explanations will be to determine whether pharmacologically enhancing TBSinduced depolarization in str. radiatum (e.g., with $\mathrm{GABA}_{\mathrm{A}}$ receptor antagonists) reduces synaptic cortactin in basal dendrites in the presence of APV.

Blocking myosin II motors with blebbistatin (Straight et al., 2003), at concentrations that do not affect baseline transmission (Rex et al., 2010), eliminated TBS effects on spine cortactin. The nonmuscle myosin light chain is phosphorylated within spines by TBS (Rex et al., 2010), an event required for actin polymerization and LTP stabilization, and is a binding partner of cortactin (Brown et al., 2010). These points strongly suggest that myosin II motors either directly transport cortactin away from synapses or promote formation of actin networks needed for such transport. A complementary mechanism is suggested by the finding that nocodazole, which accelerates depolymerization of dynamic microtubules (Vasquez et al., 1997), reduced the basal number of densely cortactin + synapses in WT slices. Combined, the results describe a dynamic subsynaptic system in which tubuledependent influx offsets a steady loss of cortactin, while events that occur during or shortly after TBS engage actomyosin motors to generate a sudden and pronounced depletion of the active (nocodazole-sensitive) pool (Fig. 6A, B). A more detailed version of this argument will require temporal parameters for the baseline cycling of the protein; notably, replacement after TBS required over $1 \mathrm{~h}$, indicating that influx is relatively slow. Beyond this lies the critical question of how intense depolarization and/or localized increases in calcium concentrations extend the influence of LTP to actomyosin networks in distant spines.

The effects of LTP along with the machinery proposed in Figure 6, $A$ and $B$, to control cortactin movement were profoundly disturbed in Fmr1 KO hippocampus: TBS did not rapidly reduce synaptic cortactin content, and nocodazole had no effect on basal concentrations. These results describe novel defects in the mutants that are plausibly related to the maintenance of spine morphology and synaptic plasticity. A search for underlying causes revealed that baseline cortactin serine phosphorylation was greatly reduced at Fmr1 KO synapses, as were increases in spine p-cortactin produced by TBS. Phosphorylation regulates cortactin's association with actomyosin motors (Lua and Low, 2005), and, indeed, coimmunoprecipation demonstrated that cortactin's association with actin was reduced in Fmr1 KO synaptoneurosomes. These findings suggest that the fundamental problem lies in the association of cortactin with upstream kinases. Coimmunoprecipitation experiments confirmed this prediction with regard to ERK1/2. Immunohistochemical analyses then established that the TBS-induced increases in synaptic p-ERK, which are robust in WT slices, are entirely absent in the mutants. These results extend descriptions of impaired ERK responses in KOs to various neuromodulators (i.e., mGluR agonists, histamine, glycine) (Hou et al., 2006; Hu et al., 2008; Kim et al., 2008; Shang et al., 2009; but see Osterweil et al., 2010) to the synaptic compartment and responses to naturalistic patterns of afferent input. The present study also establishes that the kinase is excessively phosphorylated in the spine compartment. We therefore propose that baseline disturbances to synaptic ERK1/2 activity disrupt normal links between the diffuse effects of LTP induction and the association of cortactin with actin- and tubulin-based transport machinery (Fig. 6C).

Although the normally rapid, myosin II-dependent translocation response of spine cortactin to TBS is missing in Fmr1 KO mice, a late ( $>45 \mathrm{~min}$ ) depression of spine cortactin content was observed. This delay is sufficiently long for contributions from protein translation, and there is evidence that excessive local protein synthesis occurs in the KOs (Li et al., 2001; Qin et al., 2005; Gross et al., 2010; Osterweil et al., 2010). Thus, it is possible that in these mice TBS triggers exaggerated the production of proteins that interfere with cortactin movement; an accumulation of these proteins in the spine head after TBS could lead to a delayed disruption of the constitutive positive cortactin flow toward the $\mathrm{PSD}$, and reductions in spine cortactin content, resembling effects produced by nocodazole treatment in the WTs. While speculative, this working model does make the explicit prediction that acute treatments with protein synthesis inhibitors will block the delayed loss of spine cortactin content in the mutants.

What functional consequences can be expected from the loss of cortactin mobility at Fmr1 KO spines and synapses? If the spatially distributed effects of TBS also normally occur at synapses innervated by the stimulated axons, then disturbances to cytoskeletal modifications needed for LTP consolidation are likely. We found that cortactin phosphorylation, actin associations, and movement are all disturbed in the KOs; these deficits are likely to disrupt the coordination of processes needed for normal actin and LTP consolidation in the wake of TBS. Indeed, we have found that the actin filaments that form in spines shortly after TBS are slow to stabilize, as is LTP itself, in Fmr1 KO hippocampus (Chen et al., 2010b). Beyond this, it is likely that deficits in widespread translocation responses to learning-related patterns of activity will eventually compromise the integrity of the actin networks governing spine morphology. We therefore speculate that the disturbances described in the present report (1) affect plasticity at stimulated synapses, (2) disturb adjustments pertinent to the reaction of distant connections to subsequent input, and (3) gradually degrade functional properties of spines throughout the dendritic tree.

\section{References}

Alarcon JM, Barco A, Kandel ER (2006) Capture of the late phase of longterm potentiation within and across the apical and basilar dendritic compartments of CA1 pyramidal neurons: synaptic tagging is compartment restricted. J Neurosci 26:256-264.

Amaral D, Lavenex P (2007) Hippocampal Neuroanatomy. In: The hippocampus book (Andersen P, Morris R, Amaral D, Bliss T, O’Keefe J, eds), pp 37-114. New York: Oxford UP.

Aoki C, Miko I, Oviedo H, Mikeladze-Dvali T, Alexandre L, Sweeney N, Bredt DS (2001) Electron microscopic immunocytochemical detection of PSD-95, PSD-93, SAP-102, and SAP-97 at postsynaptic, presynaptic, and nonsynaptic sites of adult and neonatal rat visual cortex. Synapse 40:239-257.

Bernard-Trifilo JA, Kramár EA, Torp R, Lin CY, Pineda EA, Lynch G, Gall CM (2005) Integrin signaling cascades are operational in adult hippocampal synapses and modulate NMDA receptor physiology. J Neurochem 93:834-849. 
Bramham CR (2008) Local protein synthesis, actin dynamics, and LTP consolidation. Curr Opin Neurobiol 18:524-531.

Brown M, Adyshev D, Bindokas V, Moitra J, Garcia JG, Dudek SM (2010) Quantitative distribution and colocalization of non-muscle myosin light chain kinase isoforms and cortactin in human lung endothelium. Microvasc Res 80:75-88.

Campbell DH, Sutherland RL, Daly RJ (1999) Signaling pathways and structural domains required for phosphorylation of EMS1/cortactin. Cancer Res 59:5376-5385.

Chen LY, Rex CS, Casale MS, Gall CM, Lynch G (2007) Changes in synaptic morphology accompany actin signaling during LTP. J Neurosci 27:5363-5372.

Chen LY, Rex CS, Sanaiha Y, Lynch G, Gall CM (2010a) Learning induces neurotrophin signaling at hippocampal synapses. Proc Natl Acad Sci U S A 107:7030-7035.

Chen LY, Rex CS, Babayan AH, Kramár EA, Lynch G, Gall CM, Lauterborn JC (2010b) Physiological activation of synaptic Rac $>$ PAK (p-21 activated kinase) signaling is defective in a mouse model of fragile $\mathrm{X}$ syndrome. J Neurosci 30:10977-10984.

Chen YK, Hsueh YP (2012) Cortactin-binding protein 2 modulates the mobility of cortactin and regulates dendritic spine formation and maintenance. J Neurosci 32:1043-1055.

Cosen-Binker LI, Kapus A (2006) Cortactin: the gray eminence of the cytoskeleton. Physiology 21:352-361.

De Roo M, Klauser P, Garcia PM, Poglia L, Muller D (2008) Spine dynamics and synapse remodeling during LTP and memory processes. Prog Brain Res 169:199-207.

Gross C, Nakamoto M, Yao X, Chan CB, Yim SY, Ye K, Warren ST, Bassell GJ (2010) Excess phosphoinositide 3-kinase subunit synthesis and activity as a novel therapeutic target in fragile X syndrome. J Neurosci 30:10624-10638.

Gu J, Zheng JQ (2009) Microtubules in dendritic spine development and plasticity. Open Neurosci J 3:128-133.

Hering H, Sheng M (2003) Activity-dependent redistribution and essential role of cortactin in dendritic spine morphogenesis. J Neurosci 23:11759-11769.

Hou L, Antion MD, Hu D, Spencer CM, Paylor R, Klann E (2006) Dynamic translational and proteasomal regulation of fragile $\mathrm{X}$ mental retardation protein controls mGluR-dependent long-term depression. Neuron 51:441-454.

Hu H, Qin Y, Bochorishvili G, Zhu Y, van Aelst L, Zhu JJ (2008) Ras signaling mechanisms underlying impaired GluR1-dependent plasticity associated with fragile X syndrome. J Neurosci 28:7847-7862.

Iki J, Inoue A, Bito H, Okabe S (2005) Bi-directional regulation of postsynaptic cortactin distribution by BDNF and NMDA receptor activity. Eur J Neurosci 22:2985-2994.

Irwin SA, Galvez R, Greenough WT (2000) Dendritic spine structural anomalies in fragile-X mental retardation syndrome. Cereb Cortex 10:1038-1044.

Irwin SA, Idupulapati M, Gilbert ME, Harris JB, Chakravarti AB, Rogers EJ, Crisostomo RA, Larsen BP, Mehta A, Alcantara CJ, Patel B, Swain RA, Weiler IJ, Oostra BA, Greenough WT (2002) Dendritic spine and dendritic field characteristics of layer $\mathrm{V}$ pyramidal neurons in the visual cortex of fragile-X knockout mice. Am J Med Genet 111:140-146.

Jaworski J, Kapitein LC, Gouveia SM, Dortland BR, Wulf PS, Grigoriev I, Camera P, Spangler SA, Di Stefano P, Demmers J, Krugers H, Defilippi P, Akhmanova A, Hoogenraad CC (2009) Dynamic microtubules regulate dendritic spine morphology and synaptic plasticity. Neuron 61:85-100.

Kim SH, Markham JA, Weiler IJ, Greenough WT (2008) Aberrant earlyphase ERK inactivation impedes neuronal function in fragile $\mathrm{X}$ syndrome. Proc Natl Acad Sci U S A 105:4429-4434.

Kirov SA, Petrak LJ, Fiala JC, Harris KM (2004) Dendritic spines disappear with chilling but proliferate excessively upon rewarming of mature hippocampus. Neuroscience 127:69-80.

Kramár EA, Lynch G (2003) Developmental and regional differences in the consolidation of long-term potentiation. Neuroscience 118:387-398.

Kruchten AE, Krueger EW, Wang Y, McNiven MA (2008) Distinct phospho-forms of cortactin differentially regulate actin polymerization and focal adhesions. Am J Physiol Cell Physiol 295:C1113-C1122.

Krucker T, Siggins GR, Halpain S (2000) Dynamic actin filaments are required for stable long-term potentiation (LTP) in area CA1 of the hippocampus. Proc Natl Acad Sci U S A 97:6856-6861.
Larson J, Lynch G (1988) Role of N-methyl-D-aspartate receptors in the induction of synaptic potentiation by burst stimulation patterned after the hippocampal theta-rhythm. Brain Res 441:111-118.

Lauterborn JC, Rex CS, Kramár E, Chen LY, Pandyarajan V, Lynch G, Gall CM (2007) Brain-derived neurotrophic factor rescues synaptic plasticity in a mouse model of fragile X syndrome. J Neurosci 27:10685-10694.

Le Clainche C, Pauly BS, Zhang CX, Engqvist-Goldstein AE, Cunningham K, Drubin DG (2007) A Hip1R-cortactin complex negatively regulates actin assembly associated with endocytosis. EMBO J 26:1199-1210.

Li B, Devidze N, Barengolts D, Prostak N, Sphicas E, Apicella AJ, Malinow R, Emamian ES (2009) NMDA receptor phosphorylation at a site affected in schizophrenia controls synaptic and behavioral plasticity. J Neurosci 29:11965-11972.

Li Z, Zhang Y, Ku L, Wilkinson KD, Warren ST, Feng Y (2001) The fragile X mental retardation protein inhibits translation via interacting with mRNA. Nucleic Acids Res 29:2276-2283.

Lua BL, Low BC (2005) Cortactin phosphorylation as a switch for actin cytoskeletal network and cell dynamics control. FEBS Lett 579:577-585.

Lynch G, Rex CS, Gall CM (2007) LTP consolidation: substrates, explanatory power, and functional significance. Neuropharmacology 52:12-23.

Lynch G, Rex CS, Chen LY, Gall CM (2008) The substrates of memory: defects, treatments, and enhancement. Eur J Pharmacol 585:2-13.

Martinez-Quiles N, Ho HY, Kirschner MW, Ramesh N, Geha RS (2004) Erk/Src phosphorylation of cortactin acts as a switch on-switch off mechanism that controls its ability to activate N-WASP. Mol Cell Biol 24:5269-5280.

Megías M, Emri Z, Freund TF, Gulyás AI (2001) Total number and distribution of inhibitory and excitatory synapses on hippocampal CA1 pyramidal cells. Neuroscience 102:527-540.

Mullins RD, Heuser JA, Pollard TD (1998) The interaction of Arp2/3 complex with actin: nucleation, high affinity pointed end capping, and formation of branching networks of filaments. Proc Natl Acad Sci U S A 95:6181-6186.

Oser M, Yamaguchi H, Mader CC, Bravo-Cordero JJ, Arias M, Chen X, Desmarais V, van Rheenen J, Koleske AJ, Condeelis J (2009) Cortactin regulates cofilin and N-WASp activities to control the stages of invadopodium assembly and maturation. J Cell Biol 186:571-587.

Osterweil EK, Krueger DD, Reinhold K, Bear MF (2010) Hypersensitivity to mGluR5 and ERK1/2 leads to excessive protein synthesis in the hippocampus of a mouse model of fragile X syndrome. J Neurosci 30:15616-15627.

Penzes P, Cahill ME, Jones KA, VanLeeuwen JE, Woolfrey KM (2011) Dendritic spine pathology in neuropsychiatric disorders. Nat Neurosci 14:285-293.

Pérez-Otaño I, Ehlers MD (2005) Homeostatic plasticity and NMDA receptor trafficking. Trends Neurosci 28:229-238.

Qin M, Kang J, Burlin TV, Jiang C, Smith CB (2005) Postadolescent changes in regional cerebral protein synthesis: an in vivo study in the FMR1 null mouse. J Neurosci 25:5087-5095.

Racz B, Weinberg RJ (2004) The subcellular organization of cortactin in hippocampus. J Neurosci 24:10310-10317.

Ramachandran B, Frey JU (2009) Interfering with the actin network and its effect on long-term potentiation and synaptic tagging in hippocampal CA1 neurons in slices in vitro. J Neurosci 29:12167-12173.

Rex CS, Chen LY, Sharma A, Liu J, Babayan AH, Gall CM, Lynch G (2009) Different Rho GTPase-dependent signaling pathways initiate sequential steps in the consolidation of long-term potentiation. J Cell Biol 186:85-97.

Rex CS, Gavin CF, Rubio MD, Kramár EA, Chen LY, Jia Y, Huganir RL, Muzyczka N, Gall CM, Miller CA, Lynch G, Rumbaugh G (2010) Myosin IIb regulates actin dynamics during synaptic plasticity and memory formation. Neuron 67:603-617.

Reymann KG, Frey JU (2007) The late maintenance of hippocampal LTP: requirements, phases, 'synaptic tagging,' 'late-associativity' and implications. Neuropharmacology 52:24-40.

Ronesi JA, Huber KM (2008) Homer interactions are necessary for metabotropic glutamate receptor-induced long-term depression and translational activation. J Neurosci 28:543-547.

Rostaing P, Real E, Siksou L, Lechaire JP, Boudier T, Boeckers TM, Gertler F, Gundelfinger ED, Triller A, Marty S (2006) Analysis of synaptic ultrastructure without fixative using high-pressure freezing and tomography. Eur J Neurosci 24:3463-3474. 
Shang Y, Wang H, Mercaldo V, Li X, Chen T, Zhuo M (2009) Fragile X mental retardation protein is required for chemically-induced longterm potentiation of the hippocampus in adult mice. J Neurochem 111:635-646.

Stäubli U, Scafidi J (1999) Time-dependent reversal of long-term potentiation in area CA1 of the freely moving rat induced by theta pulse stimulation. J Neurosci 19:8712-8719.

Straight AF, Cheung A, Limouze J, Chen I, Westwood NJ, Sellers JR, Mitchison TJ (2003) Dissecting temporal and spatial control of cytokinesis with a myosin II inhibitor. Science 299:1743-1747.

Uruno T, Liu J, Zhang P, Fan Yx, Egile C, Li R, Mueller SC, Zhan X (2001) Activation of Arp2/3 complex-mediated actin polymerization by cortactin. Nat Cell Biol 3:259-266.

Vasquez RJ, Howell B, Yvon AM, Wadsworth P, Cassimeris L (1997) Nanomolar concentrations of nocodazole alter microtubule dynamic instability in vivo and in vitro. Mol Biol Cell 8:973-985.

Walikonis RS, Jensen ON, Mann M, Provance DW Jr, Mercer JA, Kennedy MB (2000) Identification of proteins in the postsynaptic density fraction by mass spectrometry. J Neurosci 20:4069-4080.
Weaver AM, Karginov AV, Kinley AW, Weed SA, Li Y, Parsons JT, Cooper JA (2001) Cortactin promotes and stabilizes Arp2/3-induced actin filament network formation. Curr Biol 11:370-374.

Webb BA, Zhou S, Eves R, Shen L, Jia L, Mak AS (2006) Phosphorylation of cortactin by p21-activated kinase. Arch Biochem Biophys 456:183-193.

Weed SA, Karginov AV, Schafer DA, Weaver AM, Kinley AW, Cooper JA, Parsons JT (2000) Cortactin localization to sites of actin assembly in lamellipodia requires interactions with F-actin and the Arp2/3 complex. J Cell Biol 151:29-40.

Weng N, Weiler IJ, Sumis A, Berry-Kravis E, Greenough WT (2008) Earlyphase ERK activation as a biomarker for metabolic status in fragile $\mathrm{X}$ syndrome. Am J Med Genet B Neuropsychiatr Genet 147B:1253-1257.

Yang Y, Wang XB, Frerking M, Zhou Q (2008) Spine expansion and stabilization associated with long-term potentiation. J Neurosci 28:5740-5751.

zur Nedden S, Hawley S, Pentland N, Hardie DG, Doney AS, Frenguelli BG (2011) Intracellular ATP influences synaptic plasticity in area CA1 of rat hippocampus via metabolism to adenosine and activity-dependent activation of adenosine A1 receptors. J Neurosci 31:6221-6234. 